\title{
Arbitrary Steering of Multiple Particles Independently in an Electro-Osmotically Driven Microfluidic System
}

\author{
Satej Chaudhary and Benjamin Shapiro
}

\begin{abstract}
We demonstrate how to use feedback control of microflows to steer multiple particles independently in planar microfluidic systems driven by electro-osmotic actuation. This technique enables the handling of biological materials, such as cells, bacteria, DNA, and drug packets, in a hand-held format using simple and easy-to-fabricate actuators. The feedback loop consists of a vision system which identifies the positions of the particles in real-time, a control algorithm that computes the actuator (electrode) inputs based on information received from the vision system, and a set of electrodes (actuators) that create the required flow through electro-osmotic forces to steer all the particles along their desired trajectories and correct for particle position errors and thermal noise. Here, we focus on the development of control algorithms to achieve the steering of particles: vision system implementation, fabrication of devices, and experimental validation is addressed in other publications. In particular, steering of a single (yeast cell) particle has been demonstrated experimentally in our prior research and we have recently demonstrated experimental steering of three particles independently. In this paper, we develop the control algorithms for steering multiple particles independently and we validate our control techniques using simulations with realistic sources of initial position errors and thermal noise. In this study, we assume perfect measurement and actuation.
\end{abstract}

Index Terms-Electro-osmotic actuation, fluid control, microfluidics, particle steering, robust control.

\section{INTRODUCTION}

$\mathbf{M}$ ICRO-electro-mechanical systems (MEMS) are systems whose components range in size from millimeters down to micrometers. Since cells, bacteria, viruses, proteins, and DNA chains have dimensions that vary from hundreds of micrometers down to submicron scales, MEMS technologies are built at the right length scales to directly manipulate and handle such biological materials. Microfluidic systems refer to the subset of microsystems that include liquid or gas flows. Since the majority of biological entities of medical interest operate in water or in liquid buffers (DNA, proteins, and cells

Manuscript received January 14, 2005; revised November 2, 2005. Manuscript received in final form March 7, 2006. Recommended by Associate Editor S. Devasia. This work was supported in part by the National Science Foundation under Grant 0348251 and program managers Dr. R. S. Baheti and Dr. M. Burka, by the Technology Development Corporation (TEDCO), and by the Institute of Systems Research at the University of Maryland.

S. Chaudhary is with the Aerospace Engineering Department, University of Maryland, College Park, MD 20742 USA.

B. Shapiro is with the Aerospace Engineering Department, University of Maryland, College Park, MD, 20742 USA, and also with the Bio-Engineering program, University of Maryland, College Park, MD 20742 USA (e-mail: benshap@eng.umd.edu).

Digital Object Identifier 10.1109/TCST.2006.876636 function in aqueous environments), microfluidic systems are a natural choice to handle biological materials. Current microfluidic applications include microarrays for rapid analysis of DNA [1], analysis and detection of proteins [2], monitoring and analysis of cells [3], and implantable drug injection systems [4].

The ability to steer and position individual particles inside microfluidic systems is a big part of creating lab-on-a-chip or implantable miniaturized systems that can then analyze, monitor, chemically treat, and inject biological materials. Specifically, there is a need to deliver particles to localized sensors, to separate particles from each other for further testing, to steer particles into each other to test particle-to-particle interactions, to steer particles into regions of chemicals to test the response of cells, bacteria, or proteins to chemical stimuli or to drugs, and to steer particles or drug packets to external parts for drug injection and delivery.

To date, particle steering has usually been achieved in one of two ways. Particles can be steered, sorted, mixed, or separated by microfluidic plumbing architectures with valves, pumps, splitters, and mixers. For example, microchannels can be used to queue particles, the properties of these particles can then be measured, and downstream actuators can be used to shunt particles of type one to one chamber and type two to another chamber [5]. Individual particles can also be steered by optical traps or laser tweezers [6], [7]. Here, laser beams trap dielectric particles with a refractive index greater than the surrounding medium [8] and the particles then follow the imposed motion of the lasers. The main difficulty with the microfluidic plumbing method is that once the system has been built to perform a specific task, it cannot be reconfigured to perform a different task. The key drawbacks of laser tweezers are that they can only steer certain types of particles, they require delicate optics, they are expensive, and the optical system is large (about the size of a small fridge). Different particle steering approaches are appropriate for different applications. For example, in the cell clinics project at the University of Maryland, College Park [9], it is not possible to place a microfluidic plumbing architecture on top of the moving lids that open and seal the cell vials.

In this paper, we show how it is possible to combine common (electro-osmotic) actuation with feedback control to steer multiple particles independently in a microfluidic chamber. To steer particles in a planar microfluidic chamber, we will find the position of the particles in real-time using a camera and a vision algorithm, these positions will be compared to the current desired positions, a control algorithm (discussed here) will 
then compute the actuator inputs that will create a fluid flow field that will move all the particles from where they are to where they should be, and these inputs will be applied via the system actuators. This will allow us to replicate the capability of laser tweezers but with the following advantages: the entire system can be miniaturized (the microfluidics, optics, miniaturized camera, and computer processing can be squeezed into the size of a tennis ball using miniaturized cell phone cameras or into the size of match box using CMOS onchip cameras [10]); we can steer particles that laser tweezers cannot capture; and we use common electrical fluid flow actuation which means that our system is cheap. In terms of disadvantages, we note that our method does not have the ability to steer an arbitrarily large number of particles with the tens of nanometer positioning accuracy that can be achieved with laser tweezers [7].

Our multiple particle steering capability is enabled by feedback control and by the flow physics found on the microscale. Feedback is necessary to correct for particle deviations away from desired trajectories due to thermal noise, flow disturbances, and actuator errors. Microflow physics are required for the following reason. On the macroscale, fluid momentum effects create extremely complex fluid flows. It is not possible to "invert" the Navier-Stokes equations to find the right actuation that will lead to a flow field that will carry all the particles from where they are to where they should be. But on the microscale, it is easier to predict the fluid behavior. Specifically, under quasi-steady electro-osmotic actuation conditions, the Navier-Stokes equations reduce to a linear set of equations that can be effectively inverted. We can determine the necessary input voltages that will steer all the particles in the desired directions.

This paper is focused on phrasing the multiparticle steering task as a tractable control problem, on stating the appropriate control algorithms, and on validating the control algorithms using simulations that include realistic sources of thermal noise. In this study, we assume perfect measurement and actuation. In Section II, we present a brief description of a sample device, describe its mode of operation, and state the control objective. In Section III, we state the full system equations. We see that under quasi-steady actuation conditions, and using the fact that the device is of micron dimensions, the equations reduce to a simpler form which makes them amenable to robust control using classical control theory. In Section IV, we present a method to obtain the open-loop voltages for driving the particles along the desired trajectories. In addition, we also bring attention to the ill-conditioning of the open-loop voltage solutions which increases sharply as the number of particles increase. We then exploit this observation to project a theoretical limit on the number of particles that can be steered with this control method. In Section V, we show that having a large number of electrodes does not necessarily enhance our ability to control more particles and present a device with the optimal number of electrodes for the problem under investigation. In Section VI, we present a time-varying linear quadratic regulator (LQR) feedback controller to stabilize the trajectory tracking of the particles. In Section VII, we present a nonlinear feedback controller and see that it has better performance than the time-varying LQR controller. The experimental challenges of

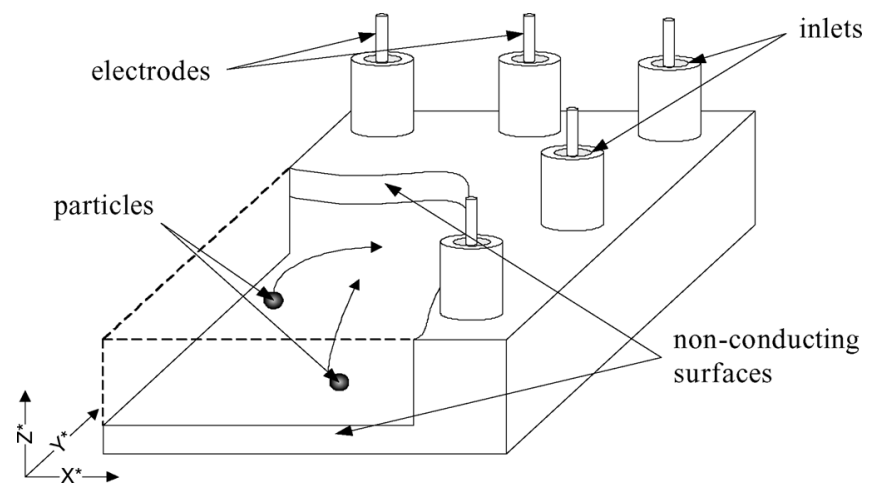

Fig. 1. Schematic of the microfluidic particle steering device. The fluid with particles is confined between two nonconducting surfaces. The actuating electrodes can create complex planar electric fields in the conducting liquid. The fluid and particles then flow along the electric field due to electrokinetic (electro-osmotic and electrophoretic) forces. Here, electrodes are shown as inserted through inlet holes in the top material into the liquid. The two nonconducting surfaces are parallel to the $x^{*} y^{*}$ plane and are located at $z^{*}= \pm h^{*} / 2$. The number and position of electrodes is a design parameter. For simulations used here, the electrodes are placed as in Fig. 4.

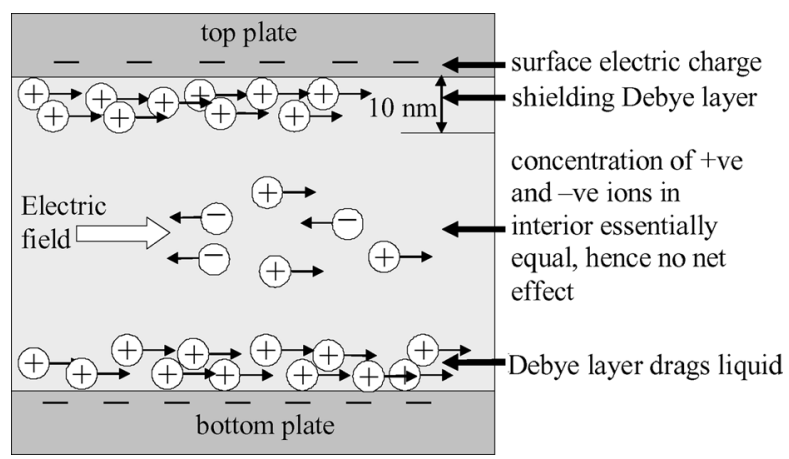

Fig. 2. Mechanism of electro-osmotic actuation of fluid at the microscale [13].

the particle steering system are discussed in other publications. In particular, [11] describes the vision system used to locate the particles in real-time and it demonstrates experimental flow control steering of a single (yeast cell) particle. Experimental steering of many particles independently is being demonstrated in current work [12].

\section{CONTROL TASK}

The control task is to steer multiple particles independently in an electroosmotically driven microfluidic system by creating an underlying fluid flow that will carry the particles along the desired trajectories. Fig. 1 shows a three-dimensional (3-D) view of a sample microfluidic system. The microfluidic chamber is enclosed by two parallel, nonconducting surfaces separated by a distance $h^{*}$ (the star denotes dimensional variables). The two nonconducting surfaces are parallel to the $x^{*} y^{*}$ plane and are located at $z^{*}= \pm h^{*} / 2$. The driving electrodes are positioned inside the channel through inlets as shown. When an electric potential is applied to the electrodes, the fluid is transported by the process of electroosmosis as illustrated in Fig. 2. Fig. 3 shows the feedback block diagram of the control system. The current positions of the particles are observed by a camera and their deviation, if any, from the desired trajectory is determined. Corrective feedback voltages are determined and applied to 


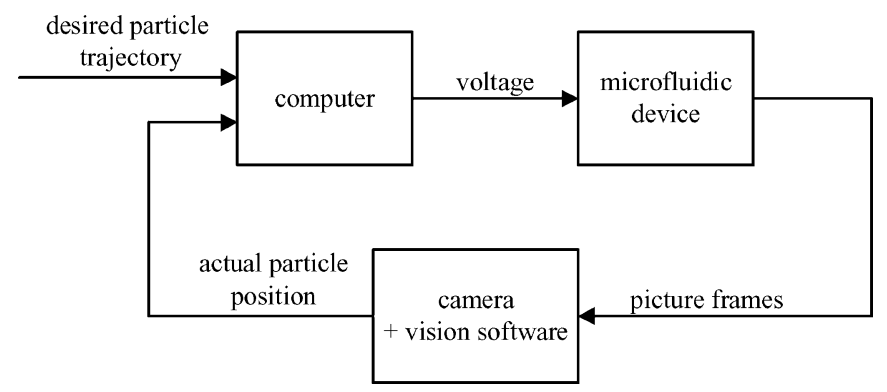

Fig. 3. Block diagram for the feedback control of multiple particles in the microfluidic device.

the electrodes to robustly steer the particles along the desired trajectories.

Electro-osmotic actuation is common in microfluidic systems [13]-[17]. When an aqueous (polar) fluid, such as water, is introduced into the microfluidic chamber, an electrical double layer, called the Debye layer, is formed at the wall/liquid interface as shown in Fig. 2. This is due to mechanisms such as acid/base reactions, ionization, ion adsorption, and ion dissolution [13]. The polarity of the charge developed depends on the material used. When an electric field is applied parallel to the wall surface, the charged double layer is displaced. The moving charged layer drags the fluid adjacent to it by viscous forces, generating an electro-osmotic flow [13]. Neutral particles are carried by this flow.

In this paper, we consider particles that are small enough to be treated as point particles but large enough so that Brownian noise is small. An appropriate size of particles satisfying this criterion is $10 \mu \mathrm{m}$. (In our experiments we use yeast cells. These cells range in diameter from 2 to $10 \mu \mathrm{m}$, are readily available from a grocery store as dry baker's yeast, are relatively robust and easy to work with, and they act as particles carried along by the flow.) Neutral particles are assumed to move with the same velocity as the surrounding fluid.

We address the following control problem: Given the desired path for every particle (position as a function of time), design a feedback controller to robustly steer the particles along these trajectories. For instance, we may require that particles 1, 2, and 5 should execute a square while particles 3 and 4 perform a figure eight.

It is also possible to consider a path planning problem. If we want to transport particles from specified initial to final locations, we can look for the optimal particle trajectories. We have not yet considered this path planning problem.

\section{Governing Fluid AND PARTICle EQUations}

In this section, we first state the governing fluid and particle equations based on the Navier-Stokes equations for the fluid dynamics and Maxwell equations for the electric fields. We assume that the particles are small enough to be treated as point particles and they flow along with the fluid. Further, we assume that the electrode voltages are varied slowly enough that the fluid flow is quasi-steady. We find that under the above assumptions, and using the fact that the device is of micron dimensions, the equations reduce to a simpler form which makes them amenable to robust control using classical control theory.

Since the minimum device length scale is well above the mean free path of the liquid molecules, the fluid flow can be accurately modeled by the continuum, incompressible, Navier-Stokes equations

$$
\begin{aligned}
\nabla \cdot \vec{V}^{*} & =0 \\
\rho^{*}\left(\frac{\partial \vec{V}^{*}}{\partial t^{*}}+\vec{V}^{*} \cdot \nabla \vec{V}^{*}\right) & =-\nabla \Psi^{*}+\eta^{*} \nabla^{2} \vec{V}^{*}
\end{aligned}
$$

where $\vec{V}^{*}\left(x^{*}, y^{*}, z^{*}\right)$ is the velocity field, $\rho^{*}$ is the fluid density (is constant), $\Psi^{*}\left(x^{*}, y^{*}, z^{*}\right)$ is the pressure, $t^{*}$ is the time, and $\eta^{*}$ is the dynamic viscosity of the fluid. Here, the star denotes dimensional quantities. Also, as the Debye layer thickness $\left(10^{-8} \mathrm{~m}\right.$ in most cases) [13] is very small compared to the chamber dimensions (length $=5 \times 10^{-4} \mathrm{~m}$, width $=5 \times 10^{-4}$ $\mathrm{m}$, height $=2 \times 10^{-5} \mathrm{~m}$ ), the boundary conditions at the walls are accurately captured by the velocity slip conditions [18]

$$
\vec{V}_{\text {wall }}^{*}\left(x^{*}, y^{*}, z^{*}\right)=\frac{\epsilon^{*} \xi^{*}}{\eta^{*}} \nabla \Phi^{*}\left(x^{*}, y^{*}\right)
$$

where $\Phi^{*}$ is the electric potential, $\epsilon^{*}$ is the permittivity of the fluid, and $\xi^{*}$ is the zeta potential at the wall. The electric potential $\Phi^{*}$ satisfies the Laplace equation [13]

$$
\nabla\left(\epsilon^{*} \nabla \Phi^{*}\right)=0
$$

with the boundary conditions of the applied voltage at the $i$ th control electrode given by

$$
\Phi^{*}\left(\partial D_{i}\right)=\gamma_{i}^{*}
$$

where $\gamma_{i}^{*}$ is the electric potential of the $i$ th electrode, and $\partial D_{i}$ denotes the electrode surface.

Our computational domain boundary is denoted by $S$ in Fig. 4. This boundary $S$ extends in the vertical direction from $z^{*}=-h^{*} / 2$ to $z^{*}=+h^{*} / 2$. It has been shown rigorously [18] that if the viscosity and surface properties are uniform and the fluid velocity at this open (computational domain) boundary $S$ is given by (6)

$$
\vec{V}_{S}^{*}=\frac{\epsilon^{*} \xi^{*}}{\eta^{*}} \nabla \Phi_{S}^{*}
$$

then the quasi-steady state solution to partial differential equations (1) and (2) in the region bounded by $S$, is simply given by

$$
\vec{V}^{*}\left(x^{*}, y^{*}, z^{*}\right)=\frac{\epsilon^{*} \xi^{*}}{\eta^{*}} \nabla \Phi^{*}\left(x^{*}, y^{*}\right) .
$$

The condition (6) is satisfied in many practical devices when the computational domain boundary is at least a few channel widths away from the electrodes. It follows, that (7) is true almost everywhere except right near the electrodes. For a more detailed discussion, please refer to [18].

We shall only consider quasi-steady fluid flow. The condition on how slowly the electrode voltages can be varied for the fluid 


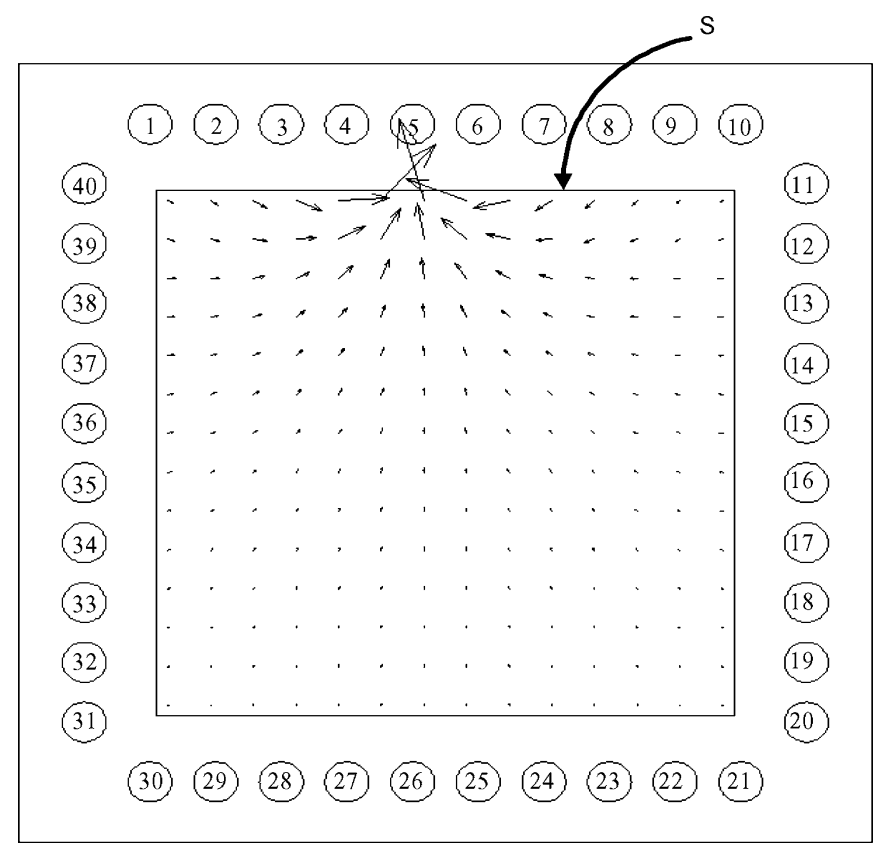

Fig. 4. Velocity field $\nabla \Phi_{5}(x, y)$ generated in the device (top view) when electrode 5 is switched to 1 voltage unit and all the other electrodes are set to 0 voltage units.

flow to qualify as quasi-steady can be estimated as follows. Consider a two-dimensional (2-D) straight channel filled with fluid which can be actuated by electroosmosis. The time taken for the electro-osmotic flow to reach $99 \%$ of steady state of (7) in response to a step voltage is given by [19]

$$
t_{s s}^{*}=0.467 \frac{h^{* 2}}{\eta^{*}} .
$$

For our devices, which are filled with water, $h^{*}=2 \times 10^{-5} \mathrm{~m}$, $\eta^{*}=0.001 \mathrm{~N} \cdot \mathrm{s} \cdot \mathrm{m}^{-2}$ so $t_{s s}^{*} \approx 2 \times 10^{-7} \mathrm{~m}$. It has been observed experimentally [19] that the flow in the intersection of two such channels may take as much as three orders of magnitude longer to reach steady state. Hence, if we vary the electric field slowly enough so that the time period of the highest frequency is much greater than $2 \times 10^{-4} \mathrm{~s}$, then for all practical purposes, the velocity of the fluid at all times is given by (7). For the remainder, we assume that the control actuation is sufficiently slow that (7) holds.

Based on (7), we can determine the particle behavior. If the particles are neutral, we can assume that they flow perfectly along with the fluid at all times. This assumption can be justified as follows. Consider a spherical particle of radius $a^{*}$ in the fluid. When the fluid flows at velocity $V_{0}^{*}$ relative to the particle, the particle experiences a drag force $F_{d}^{*}$, where

$$
F_{d}^{*}=6 \pi \eta^{*} a^{*} V_{0}^{*}
$$

is given by the classical Stokes drag law [13]. The motion of the particle as it accelerates due to $F_{d}^{*}$ can be modeled by Newton's second law as

$$
m^{*} \frac{d v^{*}}{d t^{*}}=6 \pi \eta^{*} a^{*}\left(V_{0}^{*}-v^{*}\right)
$$

where $m^{*}$ is the mass of the particle, $v^{*}$ is the velocity of the particle at any given instant, and $V_{0}^{*}-v^{*}$ is the relative velocity of the fluid with respect to the particle. The time $t_{S t}^{*}$, required for the particle to accelerate to velocity $0.999 V_{0}^{*}$, can be achieved by rearranging (10) and integrating the variable $t^{*}$ between limits 0 to $t_{S t}^{*}$ and variable $v^{*}$ between limits 0 to $0.999 V_{0}^{*}$ as

$$
\int_{0}^{0.999 V_{0}^{*}} \frac{d v^{*}}{V_{0}^{*}-v^{*}}=\frac{6 \pi \eta^{*} a^{*}}{m^{*}} \int_{0}^{t_{S t}^{*}} d t^{*}
$$

solving which, we get

$$
t_{S t}^{*}=\frac{m^{*}}{6 \pi \eta^{*} a^{*}} \ln \left(\frac{V_{0}^{*}}{0.001 V_{0}^{*}}\right) .
$$

For a particle of radius $a^{*}=10^{-5} \mathrm{~m}$ (reflecting the size of yeast cells used in our experiments) and assuming its density to be approximately equal to that of water, $\rho^{*} \approx 10^{3} \mathrm{~kg} \cdot \mathrm{m}^{-3}$, we have $t_{S t}^{*} \approx 1.5 \times 10^{-4} \mathrm{~s}$. Hence, if the average travel time of a particle along its trajectory is very large compared to $t_{S t}^{*}$, we can assume that the particles move along with the fluid.

At any time $t^{*}$, the velocity of the $j$ th particle at position ${\overrightarrow{p_{j}}}^{*}=\left(x_{j}^{*}, y_{j}^{*}\right)$ is given by

$$
\frac{d}{d t^{*}}{\overrightarrow{p_{j}}}^{*}=\vec{V}^{*}\left({\overrightarrow{p_{j}}}^{*}\right)=\frac{\epsilon^{*} \xi^{*}}{\eta^{*}} \nabla \Phi^{*}\left({\overrightarrow{p_{j}}}^{*}\right) .
$$

Equation (13) is stated for perfectly neutral particles; however, most bioparticles have a charge or acquire a surface charge when brought in contact with an aqueous medium [13]. If the particles are charged, they experience electrostatic (electrophoretic) forces; consequently, (13) is modified to [13]

$$
\frac{d}{d t^{*}} \vec{p}_{j}^{*}=(1-\beta) \frac{\epsilon^{*} \xi^{*}}{\eta^{*}} \nabla \Phi^{*}\left({\overrightarrow{p_{j}}}^{*}\right)
$$

where $\beta$ is a numerical factor which has been introduced to account for the electrostatic (electrophoretic) velocity of the particle. The coefficient $\beta$ depends on the surface charge of the particle and the thickness of the particle Debye layer in a complex way; see [13] for details. The factor $\beta$ must be identified in the experiments (by observing the particle velocity) before control implementation can proceed. Our experiments [12] verify that the particle velocity is of the form (14).

In addition to the nominal velocity, the particles exhibit a random walk or Brownian motion due to collisions with fluid molecules. The total displacement of a particle in time $d t^{*}$, incorporating displacement due to Brownian motion [20], is given by

$$
\begin{aligned}
d\left({\overrightarrow{p_{j}}}^{*}\right)=(1-\beta) \frac{\epsilon^{*} \xi^{*}}{\eta^{*}} \nabla \Phi^{*} & \left({\overrightarrow{p_{j}}}^{*}\right) d t^{*} \\
& +\sqrt{\frac{k^{*} T^{*}}{3 \pi \eta^{*} a^{*}}} \vec{\omega}(0,1) d t^{* \frac{1}{2}}
\end{aligned}
$$

where $d\left(\vec{p}_{j}\right)$ is the change in $\vec{p}_{j}^{*}$ in time $d t^{*}, k^{*}$ is the Boltzman constant, $T^{*}$ is the temperature of fluid, and $\vec{\omega}(0,1)$ is a $2 \times 1$ vector whose elements are Gaussian random variables with zero mean and covariance one. 
We now nondimensionalize (4), (5), (7), and (15) to reduce the number of physical parameters in the model. The star notation is used to discriminate between the dimensional (starred) and nondimensional (nonstarred) variables. Since the numerical values of $x^{*}, y^{*}$, and $\left|\vec{p}_{j}^{*}\right|$ are of the order of $L^{*}$, where $L^{*}$ is the length of the device, $z^{*}$ is of the order of $h^{*}$, and $\Phi^{*}, \gamma^{*}$ are of the order of $\left|\gamma_{\text {avg }}^{*}\right|$; and $\left|\vec{V}^{*}\right|$ is of the order of $\left|\vec{V}_{\text {avg }}^{*}\right|$, the relations

$$
\begin{aligned}
\Phi & =\frac{\Phi^{*}}{\left|\gamma_{\text {avg }}^{*}\right|}, \quad \vec{V}=\frac{\vec{V}^{*}}{\left|\vec{V}_{\text {avg }}^{*}\right|} \\
x & =\frac{x^{*}}{L^{*}}, \quad y=\frac{y^{*}}{L^{*}}, \quad z=\frac{z^{*}}{h^{*}} \\
\vec{p}_{j} & =\frac{\vec{p}_{j}^{*}}{L^{*}}, \quad \gamma_{i}=\frac{\gamma_{i}^{*}}{\left|\gamma_{\text {avg }}^{*}\right|} \\
\left|\vec{V}_{\text {avg }}^{*}\right| & =(1-\beta) \frac{\epsilon^{*} \xi^{*}}{\eta^{*}}\left|\nabla \Phi^{*}\right|=(1-\beta) \frac{\epsilon^{*} \xi^{*}\left|\gamma_{\text {avg }}^{*}\right|}{\eta^{*} L^{*}} \\
t & =\frac{\left|\vec{V}_{\text {avg }}^{*}\right| t^{*}}{L^{*}}=\frac{\epsilon^{*} \xi^{*}\left|\gamma_{\text {avg }}^{*}\right| t^{*}}{\eta^{*} L^{* 2}}
\end{aligned}
$$

can be used to write the equations of the system (4), (5), (7), and (15) in dimensionless variables as

$$
\nabla^{2} \Phi=0
$$

with boundary conditions

$$
\Phi\left(\partial D_{i}\right)=\gamma_{i}
$$

and

$$
\begin{aligned}
\vec{V} & =\nabla \Phi \\
d\left(\overrightarrow{p_{j}}\right) & =\vec{V}\left(\overrightarrow{p_{j}}\right) d t+c \vec{\omega}(0,1) d t^{1 / 2}
\end{aligned}
$$

where

$$
c=\sqrt{\frac{k^{*} T^{*}}{3 \pi a^{*} \epsilon^{*} \xi^{*}\left|\gamma_{\mathrm{avg}}^{*}\right|}}
$$

In our microfluidic experiments we use yeast cells which are readily available and easy to work with [12]. In general, the cell sizes range from $2 \times 10^{-6} \mathrm{~m}$ to $10^{-5} \mathrm{~m}$ in diameter [21]. For simulations in this paper, we picked values of $a^{*}=10^{-5}$ $\mathrm{m}, T^{*}=298 \mathrm{~K}, \xi^{*}=0.1 \mathrm{~V}, k^{*}=1.3807 \times 10^{-23} \mathrm{JK}^{-1}$, $\epsilon^{*}=80.2 \times 8.854 \times 10^{-12} \mathrm{C}^{2} \mathrm{~N}^{-1} \mathrm{~m}^{-2}$, and $\left|\gamma_{\text {avg }}^{*}\right|=10 \mathrm{~V}$. For these values, we find that $c \simeq 0.00025$. Hence, the term $c \vec{\omega}(0,1) d t^{1 / 2}$ is a small noise term. This completes our statement of the governing equations.

To find the open-loop control voltage we solve the equations for the nominal system, i.e., (17) with boundary conditions (18), (19), and

$$
d\left(\overrightarrow{p_{j}}\right)=\vec{V}\left(\overrightarrow{p_{j}}\right) d t
$$

which is obtained from (20) by neglecting the noise term, with initial conditions $\overrightarrow{p_{j}}(0)={\overrightarrow{p_{j}}}^{0}$, where ${\overrightarrow{p_{j}}}^{0}$ is the initial position of the $j$ th particle. We design feedback controllers that mitigate the effect of the Brownian noise and nonexact starting positions in Sections VI and VII.

We solve the PDE (17) with boundary conditions (18), using a commercial software package called FEMLAB. FEMLAB partitions the 2-D domain into triangle mesh elements and uses quadratic-Lagrangian elements to compute the solution using the finite-element method. FEMLAB then internally computes the gradient of the solution $\Phi$ to give the solutions to (19) and (21).

\section{Open-Loop Voltage And a TheOretical LiMit on THE Number OF PARTIClES THAT CAN BE STEERED}

In this section, we present a method to obtain the open-loop voltages for driving the particles along desired predetermined trajectories. In addition, we also bring attention to ill-conditioning of the open-loop voltage solutions which increases sharply as the number of particles to be steered is increased. We then exploit this observation to project a theoretical limit on the number of particles that can be steered with this control method.

The nondimensionalized equations for the nominal system, i.e., (17)-(19), are linear in $\vec{\gamma}, \Phi$, and $\vec{V}$ and they provide a static map between the voltages on the electrodes and the resulting fluid flow, so at any time $t$, the velocity field $\vec{V}$ can be expressed as a superposition of velocity fields $\nabla \Phi_{i}, i=$ $1,2, \ldots, n$, as follows:

$$
\vec{V}(x, y, \vec{\gamma})=\nabla \Phi(x, y, \vec{\gamma})=\sum_{i=1}^{n} \gamma_{i} \nabla \Phi_{i}(x, y)
$$

where $\Phi_{i}$ solves

$$
\nabla^{2} \Phi_{i}=0
$$

with boundary conditions

$$
\Phi\left(\partial D_{i}\right)=1, \quad \Phi\left(\partial D_{j}\right)=0, \quad \text { for } j \neq i
$$

Here, $n$ is the number of electrodes and $\vec{\gamma}=\left[\gamma_{1} \gamma_{2}, \ldots, \gamma_{n}\right]^{T}$ represents the vector of electrode voltages. The velocity field $\nabla \Phi_{5}(x, y)$, is shown in Fig. 4 as an example. Since $\vec{\gamma}$ and $\vec{\gamma}$ plus a constant, generate the same velocity field $\vec{V}$, we can always choose a $\vec{\gamma}$ such that a particular electrode voltage is always zero (in physical terms, one of the electrodes always acts as a ground electrode). In other words, any achievable velocity field can be expressed as a linear superposition of $n-1$ fields $\nabla \Phi_{1}, \nabla \Phi_{2}, \ldots, \nabla \Phi_{n-1}$ which constitute a linearly independent set; therefore, we can rewrite (22) as

$$
\vec{V}(x, y, \vec{\gamma})=\nabla \Phi(x, y, \vec{\gamma})=\sum_{i=1}^{n-1} \gamma_{i} \nabla \Phi_{i}(x, y)
$$




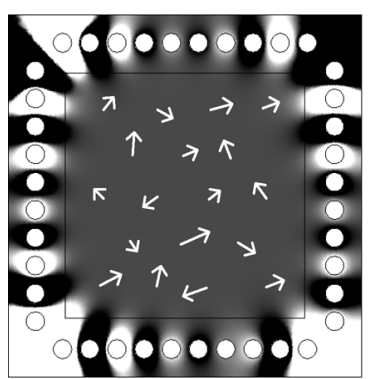

(a1)

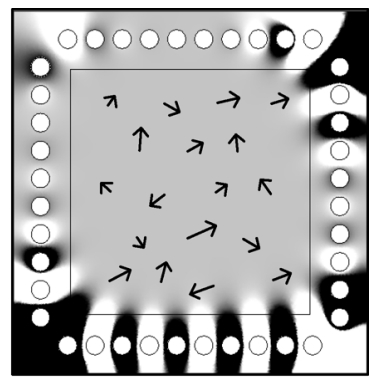

(a2)
Fig. 5. Change of $2 \%$ in the particle positions (arrow origins) and desired particle velocities (arrow directions) at 18 particle positions in the interior of the device, leads to a completely different electrode voltage distribution when (29) is used to obtain the solution. This demonstrates the ill-conditioning in the voltage solution when attempting to steer a large number of particles. Here, white represents voltage potential of positive $5 \times 10^{6}$ units and black represents voltage potential of negative $5 \times 10^{6}$ units.

If at time $t$, the particles are at positions $\overrightarrow{p_{1}}=\left(x_{1}, y_{1}\right), \overrightarrow{p_{2}}=$ $\left(x_{2}, y_{2}\right), \ldots, \overrightarrow{p_{m}}=\left(x_{m}, y_{m}\right)$, then the velocity of the $j$ th particle is given by

$$
\dot{\overrightarrow{p_{j}}}=\vec{V}\left(\overrightarrow{p_{j}}\right)=\nabla \Phi\left(\overrightarrow{p_{j}}\right)=\sum_{i=1}^{n-1} \gamma_{i} \nabla \Phi_{i}\left(\overrightarrow{p_{j}}\right) .
$$

Let ${\overrightarrow{v_{1}}}_{\text {req }},{\overrightarrow{v_{2}}}_{\text {req }}, \ldots,{\overrightarrow{v_{m}}}_{\text {req }}$ be the required particle velocities at time $t$. The objective is to combine the velocity fields $\nabla \Phi_{i}$ in the right proportions so that the fluid velocities at $\overrightarrow{p_{1}}, \overrightarrow{p_{2}}, \ldots, \overrightarrow{p_{m}}$ are as close to ${\overrightarrow{v_{1}}}_{\text {req }}, \overrightarrow{v_{2}}$ req $, \ldots, \overrightarrow{v_{m} \text { req }}$ as possible. Such a voltage vector $\vec{\gamma}_{\text {opt }}$ can be obtained by solving the least squares problem

$$
\min \left\|\vec{v}_{\text {req }}-A \vec{\gamma}_{\text {opt }}\right\|_{2}
$$

where

$$
\begin{aligned}
\vec{v}_{\text {req }} & =\left[\begin{array}{llll}
\vec{v}_{1 \text { req }} & {\overrightarrow{v_{2}}}_{\text {req }} & \ldots & \vec{v}_{m \text { req }}
\end{array}\right]^{T} \\
\vec{\gamma}_{\text {opt }} & =\left[\begin{array}{lllll}
\gamma_{1 \text { opt }} & \gamma_{2 \mathrm{opt}} & \cdots & \gamma_{n-1 \mathrm{opt}}
\end{array}\right]^{T} \\
A & =\left[\begin{array}{cccc}
\nabla \Phi_{1}\left(\overrightarrow{p_{1}}\right) & \nabla \Phi_{2}\left(\overrightarrow{p_{1}}\right) & \cdots & \nabla \Phi_{n-1}\left(\overrightarrow{p_{1}}\right) \\
\nabla \Phi_{1}\left(\overrightarrow{p_{2}}\right) & \vdots & \ldots & \vdots \\
\vdots & \vdots & \ddots & \vdots \\
\nabla \Phi_{1}\left(\overrightarrow{p_{m}}\right) & \nabla \Phi_{2}\left(\overrightarrow{p_{m}}\right) & \cdots & \nabla \Phi_{n-1}\left(\overrightarrow{p_{m}}\right)
\end{array}\right]
\end{aligned}
$$

the analytical solution of which is given by

$$
\vec{\gamma}_{\text {opt }}=\left(A^{T} A\right)^{-1} A^{T} \vec{v}_{\text {req }} .
$$

For a device with $n=40$ electrodes, when (29) is used directly to compute the voltage, it is observed that as the number of particles $m$ is increased beyond nine, the system becomes ill-conditioned, meaning smooth continuous changes in $\vec{v}_{\text {req }}$ and $\vec{p}$ produce large discontinuous changes in $\vec{\gamma}_{\text {opt }}$. Fig. 5 shows an example for 18 particles where a $2 \%$ change in $\vec{v}$ req and $\vec{p}$ entirely changes the solution voltage distribution. Moreover, the magnitude of the voltage $\vec{\gamma}_{\text {opt }}$ rises steeply with an increase in the number of particles (the voltage is of the order of
1 for 9 particles, $10^{2}$ for 12 particles, $10^{4}$ for 16 particles, and $10^{7}$ for 19 particles).

In order to understand this behavior of the solution of (27), we project the system onto its dominant singular value modes. The singular value modes provide us with an elegant framework to analyze this problem. The velocity singular value modes can be obtained in the following ways [22].

Step 1) Consider (25)

$$
\vec{V}(x, y, \vec{\gamma})=\sum_{i-1}^{n=1} \gamma_{i} \nabla \Phi_{i}(x, y)
$$

on a set of discrete $q \times q$ rectangular grid points $\overrightarrow{r_{i}}$, $i=1,2, \ldots, q^{2}$, in the domain bounded by $S$, where $q$ is chosen such that the grid resolves the velocity field sufficiently. We found that a grid with $q=50$ was adequate. Hence

$$
\vec{\Theta}=D \vec{\gamma}
$$

where

$$
\begin{aligned}
D & =\left[\begin{array}{ccc}
\nabla \Phi_{1}\left(\overrightarrow{r_{1}}\right) & \cdots & \nabla \Phi_{n-1}\left(\overrightarrow{r_{1}}\right) \\
\nabla \Phi_{1}\left(\overrightarrow{r_{2}}\right) & \cdots & \vdots \\
\vdots & \vdots & \vdots \\
\vdots & \vdots & \vdots \\
\nabla \Phi_{1}\left(\overrightarrow{r_{q^{2}}}\right) & \vdots & \nabla \Phi_{n-1}\left(\overrightarrow{r_{q^{2}}}\right)
\end{array}\right] \\
\vec{\Theta} & =\left[\begin{array}{c}
\vec{V}\left(\overrightarrow{r_{1}}\right) \\
\vec{V}\left(\overrightarrow{r_{2}}\right) \\
\vdots \\
\vdots \\
\vec{V}\left(\overrightarrow{r_{q^{2}}}\right)
\end{array}\right] \\
\vec{\gamma} & =\left[\begin{array}{c}
\gamma_{1} \\
\vdots \\
\gamma_{n-1}
\end{array}\right] .
\end{aligned}
$$

Step 2) Decompose the matrix $D$ using the singular value decomposition algorithm so that

$$
D=U \times \Omega \times W^{T}
$$

where

$$
\begin{aligned}
U & =\left[\begin{array}{llll}
U_{1} & U_{2} & , \ldots, & U_{n-1}
\end{array}\right] \\
\Omega & =\left[\begin{array}{cccc}
\sigma_{11} & 0 & 0 & 0 \\
0 & \sigma_{22} & 0 & 0 \\
0 & 0 & \ddots & 0 \\
0 & 0 & 0 & \sigma_{(n-1)(n-1)} \\
0 & 0 & 0 & 0 \\
0 & 0 & 0 & 0
\end{array}\right] \\
W & =\left[\begin{array}{llll}
W_{1} & W_{2} & , \ldots, & W_{n-1}
\end{array}\right] .
\end{aligned}
$$

Step 3) The $i$ th velocity field singular value mode is given by

$$
\overrightarrow{E_{i}}=\sum_{j=1}^{n-1} w_{j i} \nabla \Phi_{j}(x, y)
$$




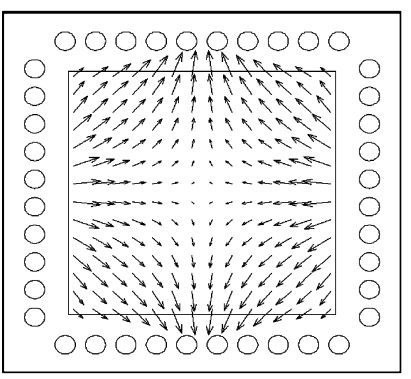

(a)

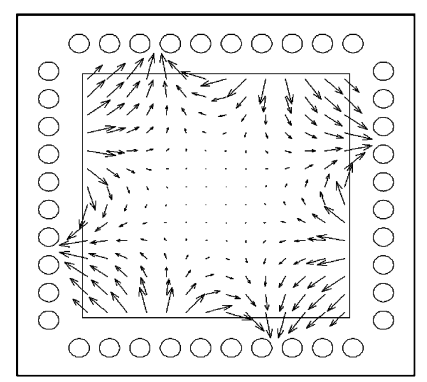

(c)

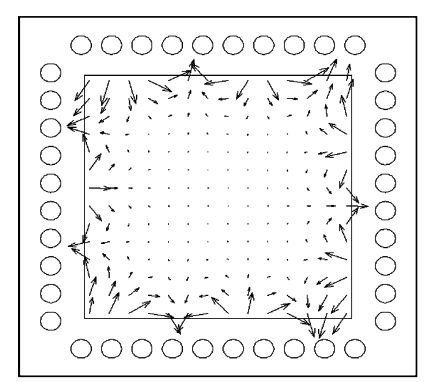

(e)

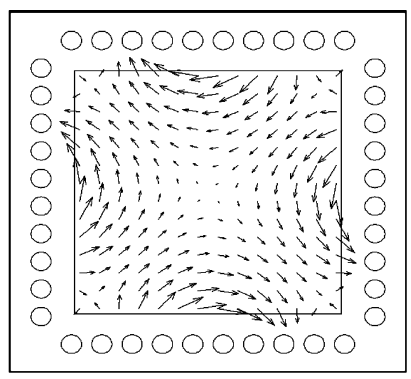

(b)

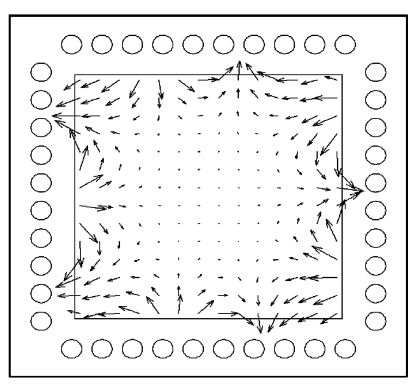

(d)

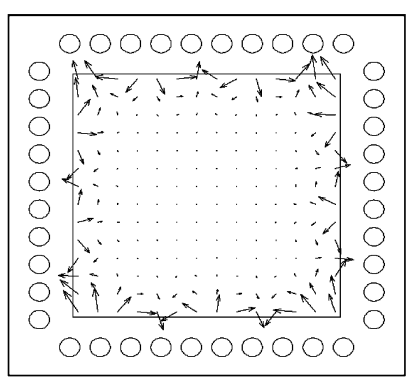

(f)
Fig. 6. Velocity field singular value modes $1,4,7,10,14$, and 18 for the 40 electrode device. (a) Velocity singular value mode 1 . (b) Velocity singular value mode 4. (c) Velocity singular value mode 7. (d) Velocity singular value mode 10. (e) Velocity singular value mode 14. (f) Velocity singular value mode 18.

where $w_{j i}$ is the element in the $j$ th row and $i$ th column of matrix $W$. The SVD enforces that the column vectors of $U$ and $W$ satisfy the following relations [23]:

$$
\begin{aligned}
\left\|U_{i}\right\|_{2} & =1 \\
\left\|W_{i}\right\|_{2} & =1 \\
D W_{i} & =\sigma_{i i} U_{i} .
\end{aligned}
$$

These relations can be physically interpreted as: application of a unit voltage vector $W_{i}$ produces a unit velocity field vector $U_{i}$ amplified by $\sigma_{i i}$. The ratio $\sigma_{i i} / \sigma_{11}$ gives the strength of the $i$ th mode of the velocity field in comparison to the 1st mode when a voltage vector of identical strength is applied to the electrodes in both cases. Fig. 6 shows the 1st, 4th, 7th, 10th, 14th, and 18th singular value modes of the velocity field for the device with $n=40$ electrodes. Fig. 7 shows the strength of the singular value modes with respect to the first mode for the same device. We see that the singular value modes beyond 25 are extremely weak in comparison to the first few singular value modes. This

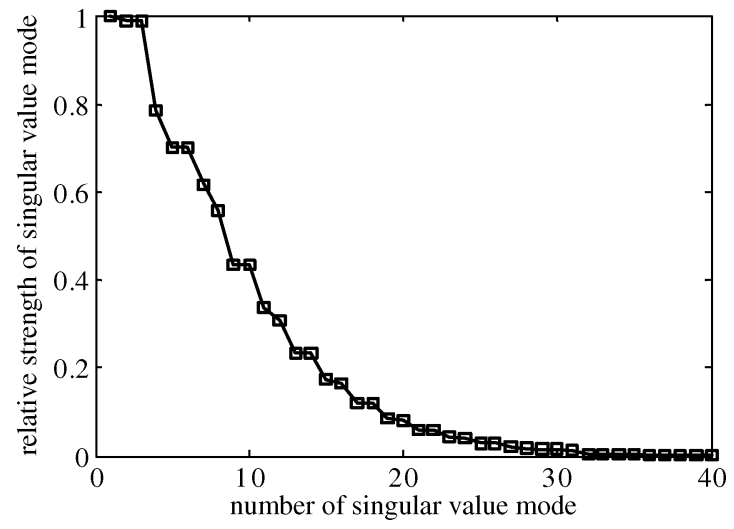

Fig. 7. Strength of the velocity field singular value modes relative to the first mode for the 40 electrode device.

means that for lower singular value modes a small voltage produces a strong velocity field, while for higher singular value modes even applying a high voltage has insignificant impact on the velocity field.

If we use only the first $e$ singular value modes to actuate the fluid, i.e., we choose a control that only includes the first $e$ singular value modes, then the velocity field at any time can be expressed as

$$
\vec{V}\left(x, y, \overrightarrow{\gamma_{E}}\right)=\sum_{i=1}^{e} \gamma_{E_{i}} \overrightarrow{E_{i}}(x, y)
$$

where $\gamma_{E_{i}}$ is the strength of the $i$ th singular value mode $\overrightarrow{\gamma_{E}}=\left[\begin{array}{llll}\gamma_{E 1} & \gamma_{E 2} & , \ldots, & \gamma_{E e}\end{array}\right]^{T}, \vec{\gamma}=W_{E} \overrightarrow{\gamma_{E}}$ and $W_{E}$ is the truncated $W$ matrix with the first e columns given by $W_{E}=\left[\begin{array}{llll}W_{1} & W_{2} & , \ldots, & W_{e}\end{array}\right]$. If at time $t$, the particles are at positions $\overrightarrow{p_{1}}=\left(x_{1}, y_{1}\right), \overrightarrow{p_{2}}=\left(x_{2}, y_{2}\right), \ldots, \overrightarrow{p_{m}}=\left(x_{m}, y_{m}\right)$, then the velocity of the $j$ th particle is given by

$$
\dot{p_{j}}=\vec{V}\left(\vec{p}_{j}\right)=\sum_{i=1}^{e} \gamma_{E_{i}} \vec{E}_{i}\left(\vec{p}_{j}\right) .
$$

Let ${\overrightarrow{v_{1}}}_{\text {req }}, \overrightarrow{v_{2}}$ req $, \ldots, \overrightarrow{v_{m} \text { req }}$ be the required particle velocities at time $t$. The objective is to combine the velocity fields $\vec{E}_{i}$ in the right proportions so that the fluid velocities at $\overrightarrow{p_{1}}, \overrightarrow{p_{2}}, \ldots, \overrightarrow{p_{m}}$ are as close to $\overrightarrow{v_{1}}$ req $, \overrightarrow{v_{2} \text { req }}, \ldots, \overrightarrow{v_{m} \text { req }}$ as possible. Such a singular value strength vector $\overrightarrow{\gamma_{E}}$ opt can be obtained by solving the transformed least squares problem

$$
\min \left\|\vec{v}_{\text {req }}-A_{E} \overrightarrow{\gamma_{E} \text { opt }}\right\|_{2}
$$

where

$$
\begin{aligned}
\vec{v}_{\text {req }} & =\left[\begin{array}{llll}
\vec{v}_{1 \text { req }} & {\overrightarrow{v_{2}}}_{\text {req }} & \ldots & {\overrightarrow{v_{m} \text { req }}}
\end{array}\right]^{T} \\
{\overrightarrow{\gamma_{\text {opt }}}} & =\left[\begin{array}{llll}
\gamma_{E_{1 \mathrm{opt}}} & \gamma_{E_{2 \mathrm{opt}}} & \ldots & \gamma_{E_{\text {eopt }}}
\end{array}\right]^{T} \\
A_{E} & =\left[\begin{array}{cccc}
\overrightarrow{E_{1}}\left(\overrightarrow{p_{1}}\right) & \overrightarrow{E_{2}}\left(\overrightarrow{p_{1}}\right) & \ldots & \overrightarrow{E_{e}}\left(\overrightarrow{p_{1}}\right) \\
\overrightarrow{E_{1}}\left(\overrightarrow{p_{2}}\right) & \vdots & \ldots & \vdots \\
\vdots & \vdots & \ddots & \vdots \\
\overrightarrow{E_{1}}\left(\overrightarrow{p_{m}}\right) & \overrightarrow{E_{2}}\left(\overrightarrow{p_{m}}\right) & \ldots & \overrightarrow{E_{e}}\left(\overrightarrow{p_{m}}\right)
\end{array}\right]
\end{aligned}
$$


the analytical solution of which is given by

$$
{\overrightarrow{\gamma_{E}}}_{\text {opt }}=\left(A_{E}^{T} A_{E}\right)^{-1} A_{E}^{T} \vec{v}_{\text {req }}
$$

The chosen applied voltage is then given by

$$
\vec{\gamma}_{\text {opt }}=W_{E} \overrightarrow{\gamma_{E}} \text { opt }
$$

When we use all the modes, i.e., $e=39$ modes to actuate the fluid, we find that the solution given by (44), is stable for $m \leq 9$ particles. When $m$ is increased beyond nine, the system becomes ill-conditioned and the magnitude of the voltage rises steeply with increase in the number of particles as mentioned before. This behavior of the solution can be explained as follows.

When the particle degrees of freedom ( $2 m$, each particle has an $x$ and a $y$ velocity) is less than the actuator degrees of freedom ( $n-1$, one electrode is ground), then (42) has multiple solutions. Of all these solutions, the least squares method picks the one with the lowest norm, i.e., the least squares method attempts to generate the required velocities at particle positions by combining the first $2 m$ velocity singular value modes as much as possible. As we increase the number of particles, progressively more velocity singular value modes are invoked. The higher singular value modes introduce large voltage components in $\vec{\gamma}_{\text {opt }}$, while having an insignificant impact on the velocity field, thus, explaining the behavior of the solution of (42) mentioned earlier.

Since we can steer only $m \leq 9$ particles, only 18 out of 39 actuation degrees of freedom are useful. These first 18 velocity singular value modes are sufficient to control 9 particles.

Fig. 8 shows snapshots of 1,4 , and 8 particles being steered using only the first 18 singular value modes. Paths of almost any shape can be achieved; however, it is not possible to steer particles that are very close together in opposite directions since this requires creating dramatically opposing flows and would require exceedingly high voltages. Fig. 9 shows the open-loop voltages of some of the electrodes for the 8 particle steering task shown in Fig. 8. Notice that the voltages vary smoothly and the magnitude of the voltage is low.

To summarize, only around 18 singular value modes of the velocity field are effectively useful for control, before the needed voltage levels become impractically high and ill-conditioned, and so we can steer up to 9 particles independently.

\section{Optimizing the Number of Electrodes In the Device}

In the last section, we saw that for the $n=40$ electrode device even though we had $n-1=39$ singular value modes only the first $e=18$ modes were useful for control, and we could independently control only around $m=9$ particles (note that 2 modes are required per particle to control the $x$ and $y$ velocities). From a practical device implementation point of view, it is important to know if arbitrary control of $m=9$ particles is possible with a device with a fewer number of electrodes. In this section, we show that a $n=20$ electrode device (5 electrodes on each side of the square) has the same amount of control authority as a $n=40$ electrode device. We can control 9 particles as effectively with a 20 -electrode device as with the 40 -electrode device.

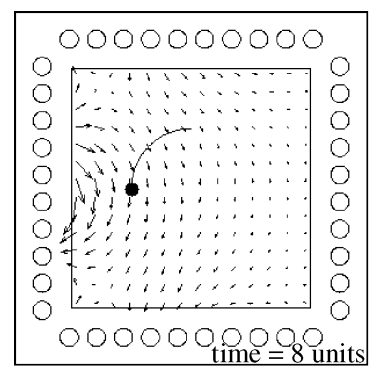

(a1)

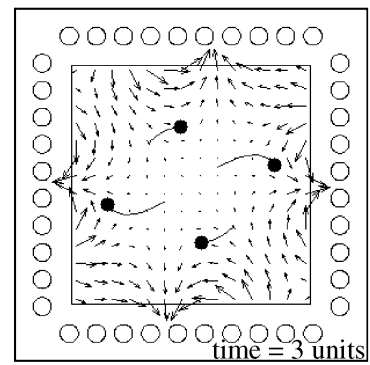

(b1)

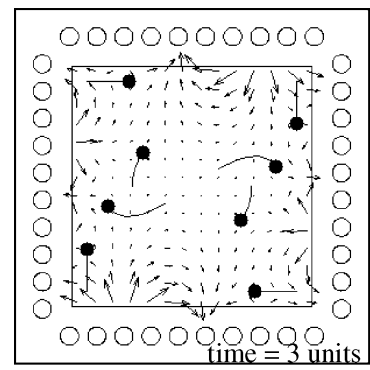

(c1)

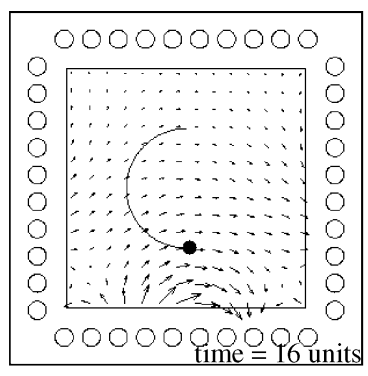

(a2)

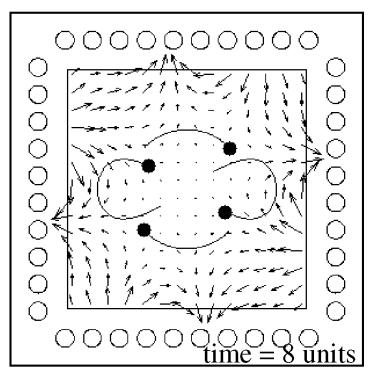

(b2)

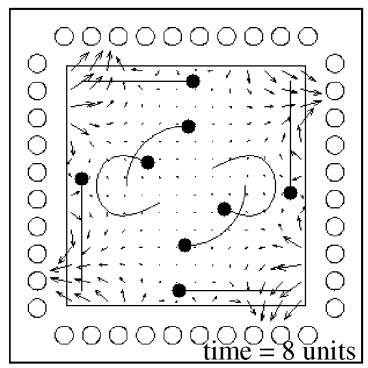

(c2)
Fig. 8. Maneuvering of 1,4 , and 8 particles successfully demonstrated in simulation for the nominal system (without noise) of the 40-electrode device. Here only the open-loop voltage is used to steer the particles.

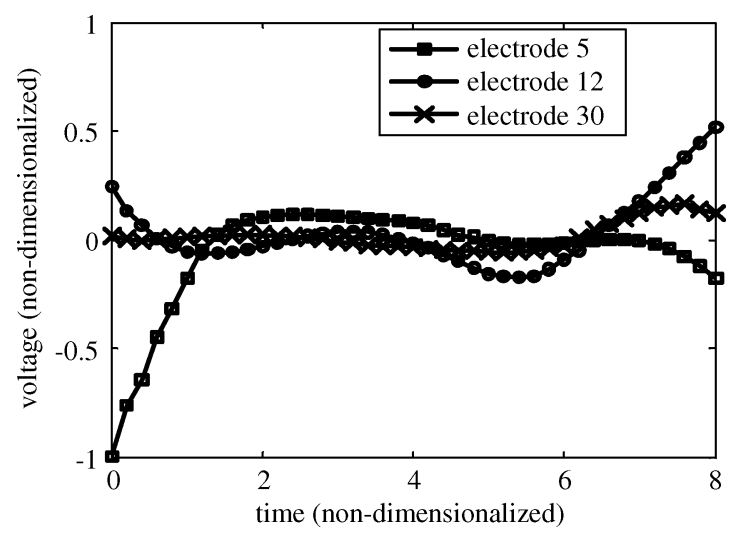

Fig. 9. Open-loop voltages for electrodes 5, 12, and 20 for the 8 particle maneuver shown in Fig. 8, subfigures $\mathrm{c} 1$ and c2, for the 40 electrode device.

Following similar procedures as outlined in the previous section, we extract the singular value modes for the 20-electrode device. The relative strength of the singular value modes of the 20-electrode device are plotted alongside that for the 40-electrode device in Fig. 10. We see that up to the 19th singular value mode, the relative strengths of the singular value modes of the 


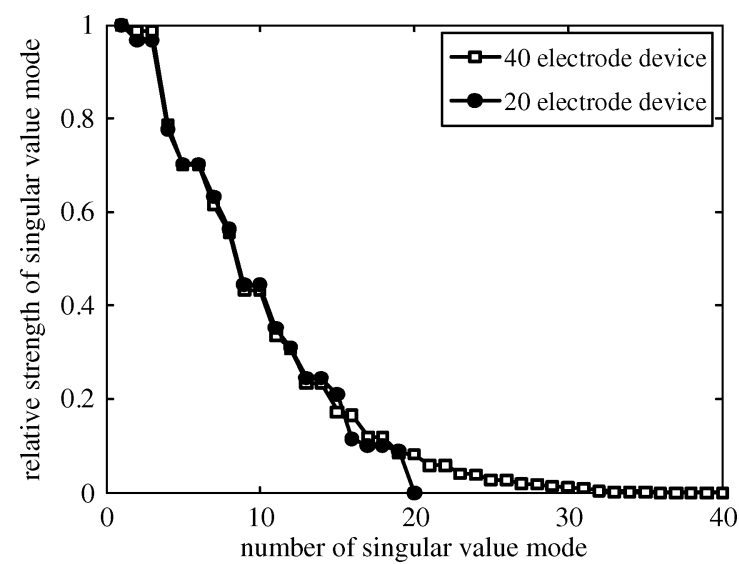

Fig. 10. Relative strength of the velocity field singular value modes for the 20 electrode device plotted alongside that for the 40 electrode device.

20 and the 40 electrode devices are almost identical. This suggests that all of the first 19 modes of the 20 electrode devices would be available for control.

Fig. 11 shows the snapshots of some of the particle steering tasks that were successfully demonstrated using the first 19 modes of the 20 electrode device. Fig. 12 shows the open-loop voltage for some of the electrodes for the eight particle steering task shown in Fig. 11. Note again, that the voltages vary smoothly and have a low magnitude. Also, since for this device all modes are available for control, it does not matter if we use velocity fields $\nabla \Phi_{1}, \nabla \Phi_{2}, \ldots, \nabla \Phi_{19}$, or singular value modes $\overrightarrow{E_{1}}, \overrightarrow{E_{2}}, \ldots, \overrightarrow{E_{19}}$, in the analysis. For the sake of keeping the discussion general in all subsequent analysis, we will work with the singular value modes.

Following similar procedures, we found that for devices with number of electrodes $n<20$ all $n-1$ singular value modes of the velocity field are available for control. Furthermore, we could perfectly control up to $m$ particles, when $2 m$ was less than $n-1$.

To summarize, for electrodes uniformly distributed along the periphery of a square, we find that we can control a maximum of around $m=9$ particles with a minimum of $n=20$ electrodes. To perfectly control $m<9$ particles, we need at least $n=$ $2 m+1$ electrodes.

\section{TIME-VARYING LQR CONTROLLER DesigN}

In an ideal situation, applying the open-loop voltage to the device will drive the particles along the corresponding trajectory. However, actuator and sensor errors, Brownian thermal fluctuations, and nonexact starting positions will cause the particles to deviate from their paths. Hence, it is necessary to apply a corrective voltage to steer the particles back to their trajectories. We have experimented with two feedback controller designs, a time-varying LQR controller and a nonlinear controller based on feedback linearization. In both cases, we design the feedback controller for the nominal system and then test its performance on a system which has realistic values of Brownian noise and particles that start from nonexact initial conditions. In this section, we present the time-varying LQR controller and also see

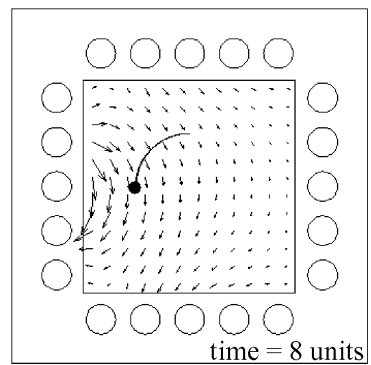

(a1)

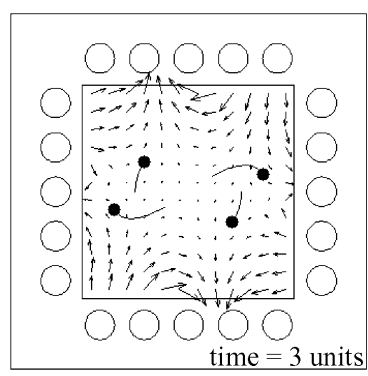

(b1)

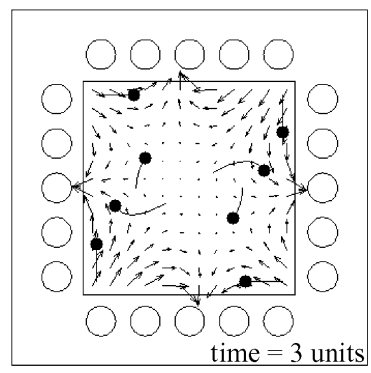

(c1)

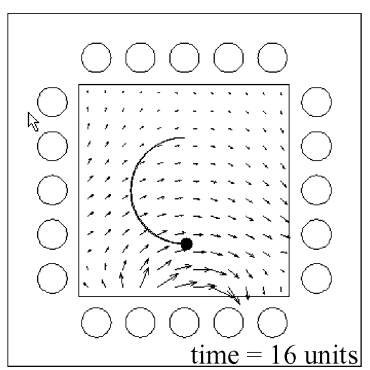

(a2)

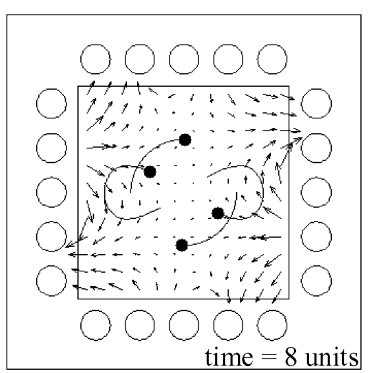

(b2)

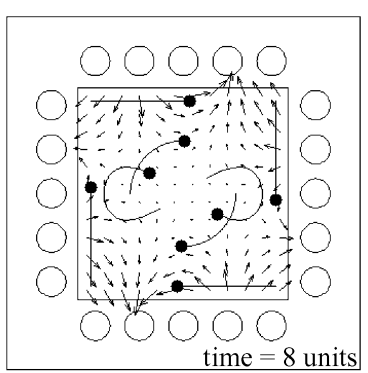

(c2)
Fig. 11. Maneuvering of 1, 4, and 8 particles successfully demonstrated in simulation for the nominal system (without noise) for the 20 electrode device. Here, only the open-loop voltage is used to steer the particles.

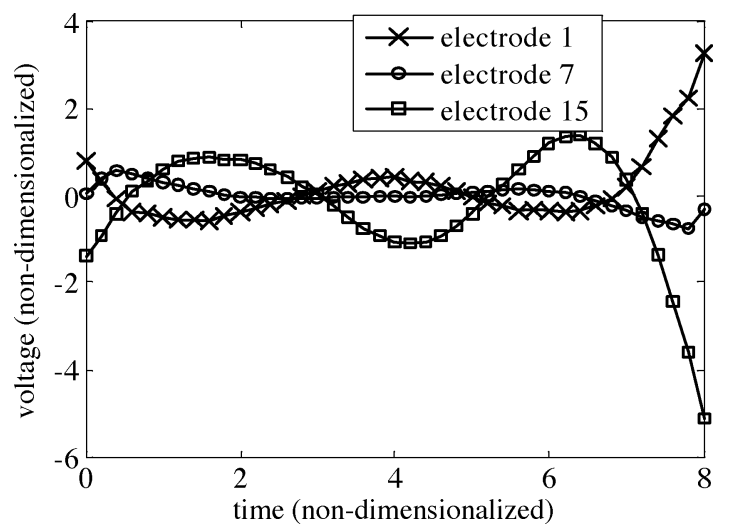

Fig. 12. Open-loop voltages for electrodes 1,7 , and 15 for the 8 particle maneuver shown in Fig. 11, subfigures (c1) and (c2), for the 20 electrode device.

its limitations. The nonlinear feedback controller is presented in the next section.

For the nominal system, if $\vec{p}(t)$ denotes the actual particle positions at time $t$, and $\overrightarrow{\gamma_{E}}(t)$ is the applied singular value mode 
strength control vector, then the actual particle dynamics is governed by

$$
\dot{\vec{p}}(t)=A_{E}(\vec{p}(t)) \overrightarrow{\gamma_{E}}(t)
$$

Let $\vec{P}(t)$ denote the desired particle trajectory and $\overrightarrow{\Gamma_{E}}(t)$ denote the open-loop control vector at time $t$, then the desired particle dynamics follow:

$$
\dot{\vec{p}}(t)=A_{E}(\vec{P}(t)) \overrightarrow{\Gamma_{E}}(t)
$$

Let $\Delta \vec{p}=\vec{p}(t)-\vec{P}(t)$ denote the particle deviation and $\Delta \overrightarrow{\gamma_{E}}=\overrightarrow{\gamma_{E}}(t)-\overrightarrow{\Gamma_{E}}(t)$ the voltage correction. Subtracting (46) and (47), assuming that $\Delta \vec{p}$ and $\Delta \overrightarrow{\gamma_{E}}$ are small and neglecting their higher order terms, we get the equations that govern the deviation of particles from the desired trajectories as

$$
\Delta \dot{\vec{p}}(t)=F(t) \Delta \vec{p}+G(t) \Delta \overrightarrow{\gamma_{E}}
$$

where

$$
\begin{aligned}
& \left.\left.F(t)=\left(\frac{\partial}{\partial \vec{p}}\left[A_{E}(\vec{p}) \overrightarrow{\gamma_{E}}\right)\right]\right)_{\left(\vec{P}(t), \overrightarrow{\Gamma_{E}}(t)\right.}\right) \\
& \left.G(t)=\left(\frac{\partial}{\partial \overrightarrow{\gamma_{E}}}\left[A_{E}(\vec{p}) \overrightarrow{\gamma_{E}}\right)\right]\right)_{\left(\vec{P}(t), \overrightarrow{\Gamma_{E}}(t)\right)} .
\end{aligned}
$$

This is a time-varying linear system. Using standard methods we design a time-varying LQR controller [24] that minimizes the cost function

$$
J=\frac{1}{2} \int_{t_{0}}^{t_{f}}\left(\Delta \vec{p}^{T} Q \Delta \vec{p}+\Delta{\overrightarrow{\gamma_{E}}}^{T} R \Delta \overrightarrow{\gamma_{E}}\right)
$$

to drive $\Delta \vec{p}$ to zero. Here, $Q$ and $R$ are positive definite matrices and are chosen to ensure that we have acceptable levels of $\Delta \vec{p}$ and $\Delta \overrightarrow{\gamma_{E}}$ for all time.

In the simulation to test the LQR controller, the particles were started 0.025 units away from the exact initial positions and the Brownian noise parameter $c$ for the system (20) was set to 0.00025 , as corresponds to a particle size of $10 \mu \mathrm{m}(10 \times$ $10^{-6} \mathrm{~m}$ ), the time step size was chosen to be 0.1 and both matrices $Q$ and $R$, were chosen to be identity. We found that the time-varying LQR controller failed to be effective for maneuvers involving more than two particles. This is because as we increase the number of particles, the velocity field becomes progressively more complex and the particle dynamics fall outside the linearization range.

\section{Nonlinear FeEdBack Controller Design}

In this section, we present a nonlinear feedback controller based on the technique of feedback linearization. We also see the advantages of this controller over the LQR controller presented in the last section.

The actual particle dynamics is governed by

$$
\dot{\vec{p}}(t)=A_{E}(\vec{p}(t)) \overrightarrow{\gamma_{E}}(t)
$$

As in (40), we choose to apply control voltages that correspond to the first $E$ singular value modes only, hence, in this equation there is no singular value truncation error. We use the feedback law [25]

$$
\overrightarrow{\gamma_{E}}(t)=A_{E}^{\dagger}(\vec{p}(t))(-k \Delta \vec{p}(t)+\dot{\vec{P}}(t))
$$

where $A_{E}^{\dagger}(\vec{p})=\left(A_{E}^{T}(\vec{p}) A_{E}(\vec{p})\right)^{-1} A_{E}^{T}(\vec{p})$ is the pseudoinverse of $A_{E}(\vec{p})$ [23] and $k$ is a positive scalar. When the number of columns of $A_{E}$ are greater than the number of its rows (which is valid in our case, since we always keep $2 m+$ $1<n$ as mentioned in Section $\mathrm{V}$ ), and $A_{E}$ has full rank (as is the case if particles do not overlap) then $A_{E}(\vec{p}) A_{E}^{\dagger}(\vec{p})=I$; consequently, the system equations (52) are transformed to

$$
\dot{\vec{p}}(t)=\dot{\vec{P}}(t)-k \Delta \vec{p}(t)
$$

or

$$
\Delta \dot{\vec{p}}(t)=-k \Delta \vec{p}(t)
$$

and we can see that this feedback law will drive the deviation to zero at an exponential rate. This nonlinear controller was successfully used for robustly steering 1,4 , and 8 particle trajectories as shown in Fig. 13. In the simulation to test the nonlinear controller, the particles were started 0.025 units away from the exact initial positions and the Brownian noise parameter $c$ for the system (20) was set to 0.00025 , as corresponds to a particle size of $10 \mu \mathrm{m}\left(10 \times 10^{-6} \mathrm{~m}\right)$, the time step size was chosen to be 0.1 and $k$ was chosen to be 2 . Fig. 14 shows the voltages of some of the electrodes for the eight particle maneuver. The steady-state error in $x$ and $y$ particle positions for this case, was found to be approximately 0.005 . This means that, from (16), for a device of size $L^{*}=500 \mu \mathrm{m}$, the error in particle positions would be approximately $2.5 \mu \mathrm{m}$.

The nonlinear feedback controller successfully tracks particles even in presence of significant Brownian noise. Fig. 15 shows a simulation for the four particle control task when the Brownian noise parameter $c$ was increased to 0.03 . (Note that even if the particle is only $1 \mu \mathrm{m}$ big $\left(10^{-6} \mathrm{~m}\right)$ the smallest size that can be seen optically then $c=0.0008$. Here, the Brownian noise coefficient was chosen to be around 38 times higher than this value.) In this case, the steady-state error in $x$ and $y$ particle positions is 0.04 . This means that, from (16), for a device of size $L^{*}=500 \mu \mathrm{m}$, the error in particle positions would be approximately $20 \mu \mathrm{m}$. We have used this controller to successfully steer up to three particles in our experimental devices [12].

\section{CONCLUSION}

This paper shows how to combine feedback control and microfluidics to steer multiple particles independently in microfluidic systems: an issue that is important for the handling of biological materials in miniaturized systems. We consider planar microfluidic devices driven by common electro-osmotic actuation, and we simplify the fluid Navier-Stokes equations, the Debye layer fluid slip boundary conditions, and the Maxwell electrical equations to phrase the particle steering question as a tractable control problem. The open-loop part of the control 


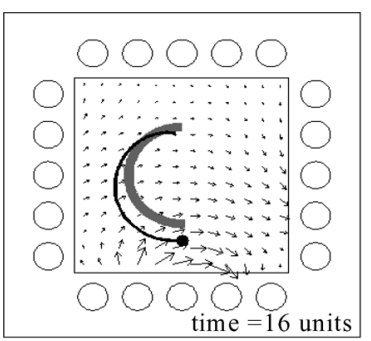

(a1)

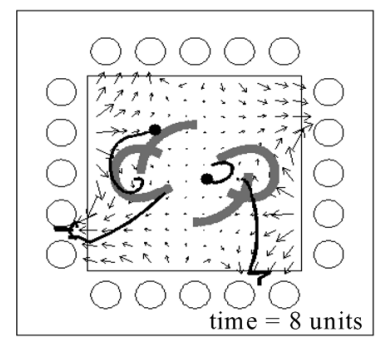

(b1)

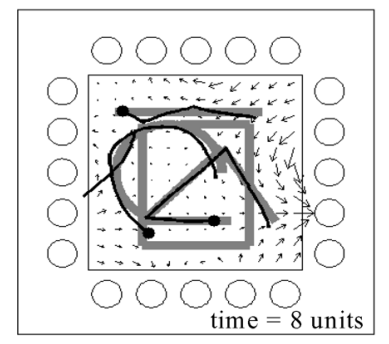

(c1)

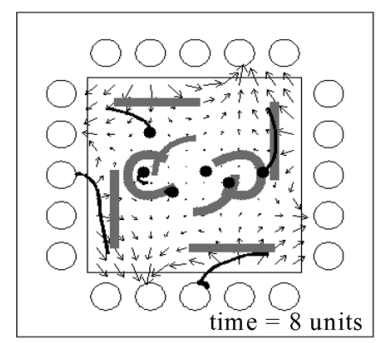

(d1)

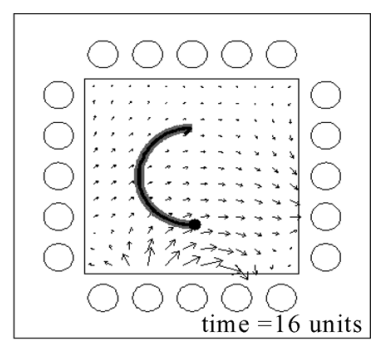

(a2)

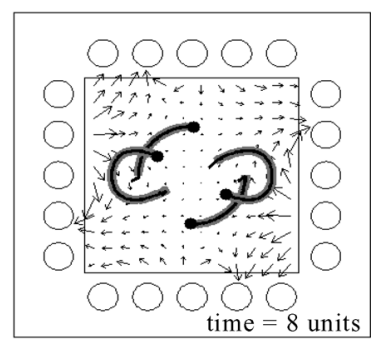

(b2)

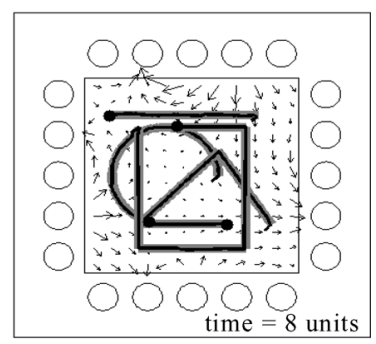

(c2)

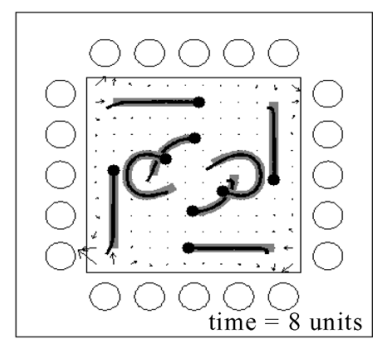

(d2)
Fig. 13. Left-hand-side figures show the deviation of the particles from the path when only the open-loop voltage is applied. The right-hand side shows the trajectories of the particles when the nonlinear controller based on feedback linearization is used. In the simulation the particles start from nonexact initial conditions and the system has Brownian noise incorporated in it. The thick grey lines show desired trajectories of the particles and the thin black lines indicate the actual trajectories of the particles. The actual and the desired paths for the nonlinear controller in the right panels are so close that the grey and black curves overlay each other.

problem is solved using a least squares method; the closed loop noise rejection portion is addressed either by a feedback linearization technique or an $\mathrm{LQR}$ technique.

The steering of multiple particles independently is demonstrated in numerical simulations that include realistic amounts of initial position errors and thermal noise. The steering of a single yeast cell has been demonstrated experimentally in our prior research [11], and the experimental steering of multiple particles independently is being addressed in a current publication [12]. We note that this application is a good example of how control can enable new, as opposed to simply improved, capabilities in MEMS. Feedback control allows particle steering

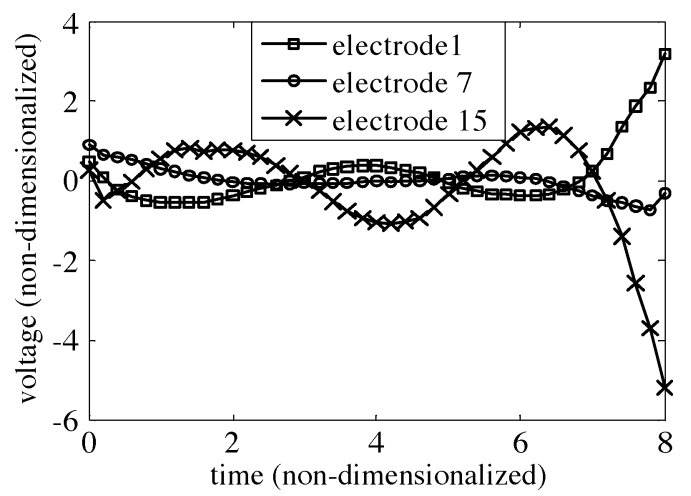

Fig. 14. Total voltages for electrodes 1,7 , and 15 for the 8 particle maneuver regulated by the nonlinear controller shown in Fig. 13, subfigure c2.

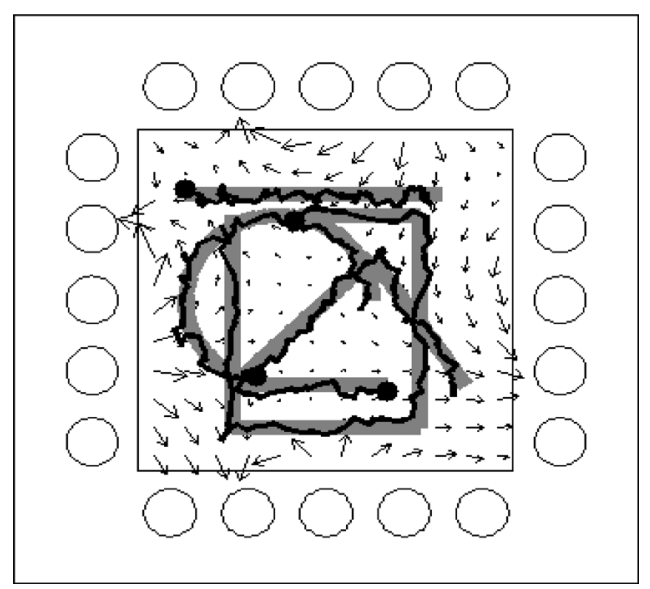

Fig. 15. Nonlinear feedback controller is successful in tracking multiple particles even in presence of substantial Brownian noise. Here the Brownian noise parameter was set to 0.03 (note the rough particle trajectories). The thick grey lines show desired trajectories of the particles and the thin black lines indicate the actual trajectories of the particles.

capabilities in microfluidic devices with common, easy to fabricate, and miniaturized actuators. It replaces the need for bulky and expensive laser tweezer equipment by a feedback control system and a vision system that can be integrated on chip, hence, permitting laser tweezer capabilities in a hand-held format.

\section{REFERENCES}

[1] M. A. Northrup, B. Benett, D. Hadley, P. Landre, S. Lehew, J. Richards, and P. Stratton, "A miniature analytical instrument for nucleic acids based on micromachined silicon reaction chambers," Anal. Chem., vol. 70, no. 5, pp. 918-922, Mar. 1998.

[2] D. Figeys, "Adapting arrays and lab-on-a-chip technology for proteomics," Proteomics, vol. 2, no. 4, pp. 373-382, Apr. 2002.

[3] J. Seo, C. Ionescu-Zanetti, J. Diamond, R. Lal, and L. P. Lee, "Integrated multiple patch-clamp array chip via lateral cell trapping junctions," Appl. Phys. Lett., vol. 84, no. 11, pp. 1973-1975, Nov. 2004.

[4] G. Spera, "Implantable pumps improve drug deliver, strengthen weak hearts," Med. Dev. Diagnostic Ind. Mag., Sep. 1997.

[5] A. Y. Fu, H. Chou, C. Spence, F. H. Arnold, and S. R. Quake, "An integrated microfabricated cell sorter," Anal. Chem., vol. 74, no. 11, pp. 2451-2457, Nov. 2002.

[6] S. H. Chi-Kuang, H. Yin-Chieh, and C. P. Chin, "Cell manipulation by use of diamond microparticles as handles of optical tweezers," J. Opt. Soc. Amer. B, vol. 18, no. 10, pp. 1483-1489, Oct. 2001.

[7] J. E. Curtis, B. A. Koss, and D. G. Grier, "Dynamic holographic optical tweezers," Opt. Commun., vol. 207, no. 1-6, pp. 169-175, Apr. 2002.

[8] A. Ashkin, "History of optical trapping and manipulation of small-neutral particles, atoms and molecules," IEEE J. Sel. Topics Quantum Electron., vol. 6, no. 6, pp. 841-856, Nov./Dec. 2000. 
[9] Y. Liu, N. M. Nelson, P. Abshire, and E. Smela, "Biolab-on-a-chip for capturing, culturing, and in-situ investigation of living cells," in Proc. Micro Total Anal. Syst., 8th Int. Conf. Miniaturized Syst. Chem. Life Sci., 2004, pp. 584-585.

[10] E. R. Fossum, "CMOS image sensors: electronic camera-on-a-chip," IEEE Trans. Electron Devices, vol. 44, no. 10, pp. 1689-1698, Oct. 1997.

[11] M. Armani, S. Chaudhary, R. Probst, and B. Shapiro, "Using feedback control and micro-fluidics to steer individual particles," in Proc. IEEE 18th Int. Conf. Microelectromech. Syst., 2005, to be published.

[12] M. A. Armani, S. V. Chaudhary, R. Probst, and B. Shapiro, "Using feedback control of micro-fluidics to independently steer multiple particles," J. Microelectromech. Syst., to be published.

[13] R. F. Probstein, Physiochemical Hydrodynamics. New York: Wiley, 1994.

[14] P. C. H. Li and D. J. Harrison, "Transport, manipulation, and reaction of biological cells on-chip using electrokinetic effects," Anal. Chem., vol. 69, no. 8, pp. 1564-1568, Aug. 1997.

[15] P. A. Auroux, D. Iossifidis, D. R. Reyes, and A. Manz, "Micro total analysis systems. 2. Anaytical standard operations and applications," Anal. Chem., vol. 74, no. 12, pp. 2637-2652, Dec. 2002.

[16] A. Y. Fu, C. Spence, A. Scherer, F. H. Arnold, and S. R. Quake, "An microfabricated fluoroscence-activated cell sorter," Nature Biotechnol., vol. 17, no. 11, pp. 1109-1111, Nov. 1999.

[17] A. Manz, C. S. Effenhauser, N. Burggraf, D. J. Harrison, K. Seiler, and K. Flur, "Electro-osmotic pumping and electrophoretic separations for miniaturized chemical analysis systems," J. Micromech. Microeng., vol. 4, no. 4, pp. 257-265, Dec. 1994.

[18] E. B. Cummins, S. K. Griffiths, R. H. Nilson, and P. H. Paul, "Conditions for similitude between the fluid velocity and electric field in electroosmotic flow," Anal. Chem., vol. 72, no. 11, pp. 2526-2532, Nov. 2000.

[19] N. A. Patankar and H. H. Hu, "Numerical simulation of electro-osmotic flow," Anal. Chem., vol. 70, no. 9, pp. 1870-1881, Sep. 1998.

[20] D. T. Gillespie, "The mathematics of Brownian motion and Johnson noise," Amer. J. Phys., vol. 64, no. 3, pp. 225-240, Mar. 1996.

[21] Mc Graw-Hill Encyclopedia of Science and Technology, 8th ed. New York: McGraw-Hill, 1997.

[22] P. C. Hansen, Rank-Deficient and Discrete Ill-Posed Problems: Numerical Aspects of Linear Inversion. Philadelphia, PA: SIAM, 1998.

[23] G. Strang, Linear Algebra and It's Applications. Pacific Grove, CA: Brooks/Cole, 1988.
[24] A. E. Bryson and Y. C. Ho, Applied Optimal Control. New York: Taylor and Francis, 2004.

[25] H. K. Khalil, Nonlinear Systems. Upper Saddle River, NJ: PrenticeHall, 2002.

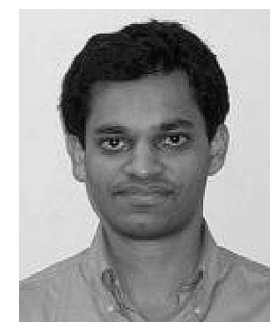

Satej Chaudhary was born in Bombay, India, in 1978. He received the B.Tech. degree in aerospace engineering from the Indian Institute of Technology, Bombay, India, in 2001, and is currently pursuing the $\mathrm{Ph} . \mathrm{D}$. degree in modeling and control of microfluidic systems at the University of Maryland, College Park.

His research interests include MEMS, control theory, and robust control.

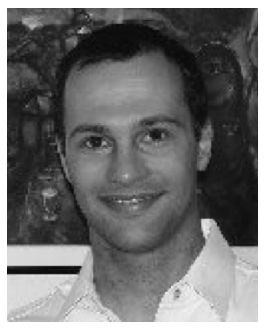

Benjamin Shapiro was born in Jerusalem, Israel, in 1973. He received the B.S. degree in aerospace engineering from the Georgia Institute of Technology, Atlanta, in 1995, and the Ph.D. degree in control and dynamical systems from the California Institute of Technology, Pasadena, in 1999.

In 2000, he joined the Department of Aerospace Engineering at the University of Maryland, College Park, as an Assistant Professor. His main interests are in modeling and control of microsystems, with a primary focus on modeling and control of microfluidic systems, for biochemical applications.

He is part of the interdisciplinary Small Smart Systems Center at the University of Maryland and holds joint appointments with the Bioengineering graduate program and the Applied Math and Scientific Computation Program. He was the organizer of the March 2004 National Science Foundation workshop on "Control and System Integration of Micro and Nanoscale Systems." 